\title{
Age-related decline in leg-extensor power development in single- versus multi-joint movements
}

\author{
Stijn Van Driessche ${ }^{\mathrm{a}, *}$, Evelien Van Roie ${ }^{\mathrm{a}}$, Benedicte Vanwanseele ${ }^{\mathrm{b}}$, Marc Van Leemputte ${ }^{\mathrm{c}}$, \\ Christophe Delecluse ${ }^{a}$
}

${ }^{\text {a }}$ KU Leuven, Department of Movement Sciences, Physical Activity, Sports and Health Research Group, Belgium

${ }^{\mathrm{b}} \mathrm{KU}$ Leuven, Department of Movement Sciences, Human Movement Biomechanics Research Group, Belgium

${ }^{\mathrm{c}}$ KU Leuven, Department of Movement Sciences, Exercise Physiology Research Group, Belgium

\section{A R T I C L E I N F O}

\section{Keywords:}

Physical function

Rapid strength

Sarcopenia

Functional performance

\begin{abstract}
A B S T R A C T
Rapid muscle characteristics, such as the rate of power development (RPD), are shown to decline more than maximal muscle characteristics during ageing in single-joint actions. However, functional disability is mainly the result of multi-joint lower limb failure. The complex activation patterns inherent to multi-joint actions and the deteriorating effect of age on that neural drive suggest a larger effect of age on RPD multi-joint. Yet, this is the first study that compared multi- with single-joint leg extension tests in terms of RPD across the adult life span and assessed its transferability to functional performance.

96 healthy adults ( $\sigma^{\prime} 49$, $\subsetneq 47$, age $=20-69$ years) performed dynamic single-joint knee-extension tests on a Biodex System 3 dynamometer and multi-joint leg-extension tests on a custom-made device at low, moderate and high speed. Peak power (Ppeak) was calculated as the highest value of the power-time curve and RPD as the linear slope till isokinetic speed was reached. Functional performance was tested using squat jump height.

RPD showed greater age-related declines in multi-joint $(-1.92 \% /$ year $)$ versus single-joint $(-1.42 \% /$ year $)$ actions, which is in contrast with the finding of Ppeak ( $-0.77 \%$ vs. $-1.04 \%$ year). Squat jump height was more strongly associated with RPD multi-joint than single-joint ( $r=0.77-0.82$ vs. $0.44-0.61)$.

These results show greater age-related declines of RPD multi-joint versus single-joint and demonstrate its functional relevance. We believe that this finding may be of high importance for the detection and prevention of functional disability during ageing.
\end{abstract}

\section{Introduction}

One of the major problems in our ageing and graying society is the age-related loss of independence, resulting in enormous health care costs. This loss of independence, manifested by a decreased physical performance and an increased fall risk, is a consequence of the effects of ageing on neuromuscular function (Kennis et al., 2014; Madigan and Lloyd, 2005; Aagaard et al., 2010). Therefore, optimized screening and prevention methods for this neuromuscular decline are highly needed.

The majority of screening methods in research and clinical practice focused on maximal muscle strength or maximal muscle power (Goodpaster et al., 2006; Kostka, 2005; Yamauchi et al., 2009; Macaluso and De Vito, 2003). Maximal power production deteriorates more during ageing compared to maximal strength (Lanza et al., 1985; Skelton et al., 1994). Moreover, muscle power is a better determinant of functional capacity compared to muscle strength (Cuoco et al., 2004; Foldvari et al., 2000), which suggest that dynamic tests to evaluate power are preferred over isometric tests for screening of neuromuscular function. However, neither maximal strength nor maximal power take into account the time needed to be developed (i.e. $>300 \mathrm{~ms}$ ). In other words, these variables may not be representative for the initial phase of very quick movements, such as the prevention of a fall after stumbling (Madigan and Lloyd, 2005; Pijnappels et al., 2005).

This initial phase of very quick movements is represented by parameters such as the rate of force (RFD) or power development (RPD). $\mathrm{RFD}$, i.e. the ability to produce force rapidly, declines to a greater extent during ageing and is more strongly related to functional daily tasks and fall risk than maximal strength (Häkkinen et al., 1998; Thompson et al., 2013; Clark et al., 2013; Izquierdo et al., 1999). In line with these findings, a recent study in our lab demonstrated that the age-related

\footnotetext{
Abbreviations:peak power (Ppeak), rate of power development (RPD)

* Corresponding author at: Physical Activity, Sports and Health Research Group, Department of Movement Sciences, Faculty of Movement and Rehabilitation Sciences, KU Leuven, Tervuursevest 101, 3001 Leuven, Belgium.

E-mail address: stijn.vandriessche@kuleuven.be (S. Van Driessche).
} 
declines in RPD exceeded the declines in maximal power (Ppeak) performed in dynamic contractions (Van Driessche et al., 2018a). Conjointly, we can conclude that older adults have even greater difficulty in developing strength or power rapidly than in reaching high peak values. However, most studies were limited to isolated single-joint movements, which may not be representative for most daily life activities that include multi-joint movements (Azegami et al., 2007).

Multi-joint movements primarily differ from single-joint movements through their complex neural activation and coordination patterns of agonist and antagonist/synergist muscles. This neural activation capacity, which is crucial for rapid force production in older adults (Klass et al., 1985), is detrimentally affected by ageing (Billot et al., 2014). Therefore, ageing may have a different impact on rapid force production in multi- versus single-joint movements. To date, few studies have focused on rapid force production in multi-joint actions. These studies were either limited to isometric tests or did not compare multi-joint to single-joint tests within the same cohort (Izquierdo et al., 1999; Allison et al., 2013; Thompson et al., 2018).

Therefore, this study investigated the effect of ageing on maximal and rapid muscle characteristics in dynamic single- and multi-joint actions across the adult life span. In addition, associations with a functional movement were evaluated. We hypothesized that: 1) RPD declines more than Ppeak in both test methods, 2) RPD declines more in multi-joint compared to single-joint movements and 3) multi-joint RPD is more strongly related to a functional movement than single-joint RPD.

\section{Methods}

\subsection{Subjects}

Men and women between 20 and 70 years old $\left(n=10 \sigma^{n}\right.$ and $10 \%$ per decade) were recruited through advertisements and oral communications. Subjects completed a short medical history and activity questionnaire and were excluded in case of a cardiovascular disease or acute thrombosis, recent surgery, neuromuscular disease, infection or fever, diabetes or pregnancy and systematic strength or endurance training (i.e. progressive increases in volume and/or intensity) in the prior 6 months. Occasional engagement in physical activity, such as cycling, walking and running was allowed. In total, 96 healthy subjects $\left(\sigma^{\prime} 49\right.$, $q$ 47) aged between 20 and 69 years volunteered and their data were included for all analyses. All subjects provided written informed consent. The study was approved by the University's Human Ethics Committee in accordance with the declaration of Helsinki.

\subsection{Design}

This study had a cross-sectional design to reveal the effect of age, sex, speed and test method on upper leg neuromuscular function. Subjects performed two test sessions separated by a rest day to avoid fatigue. In the first session, familiarization with the multi-joint protocol was performed as a warming-up, followed by the single-joint protocol. The second session was performed at the same time of day as session one and led by the same investigator. Participants performed a warming-up on a bike ergometer at a self-determined submaximal resistance for $10 \mathrm{~min}$, before performing the functional performance tests and the multi-joint protocol.

\subsection{Single-joint testing}

Neuromuscular function of the knee extensors was measured using a standardized protocol on a Biodex Medical System $3^{\circledast}$ dynamometer with a sampling rate of $100 \mathrm{~Hz}$ (Biodex Medical Systems, Shirley, New York, USA). Measurements were performed unilaterally on the right side, in a seated position on a vertically and horizontally adjustable backward-inclined $\left(5^{\circ}\right)$ chair. Range of motion was set from a knee joint angle of $90^{\circ}$ to $160^{\circ}$, with a fully extended leg corresponding to a knee angle of $180^{\circ}$. The upper leg, hips and shoulders were stabilized with safety belts. The rotational axis of the dynamometer was aligned with the transversal knee-joint axis and connected to the distal end of the tibia with a length-adjustable rigid lever arm. After warming-up, subjects performed four isometric knee-extension tests at $90^{\circ}$ knee joint angle. They were instructed to push as hard as possible for $5 \mathrm{~s}$, separated by a 20 -second rest period. Next, after at least 2 min of rest, subjects performed a series of three consecutive maximal isokinetic knee extension movements at slow, moderate and high speed (i.e. 60, 180 and $300^{\circ} / \mathrm{s}$ ). During each dynamic test, the subjects were clearly instructed by the test leader to perform the tests as fast and as hard as possible. The rest interval was $30 \mathrm{~s}$ between the three conditions.

\subsection{Multi-joint testing}

A modified isokinetic version of the Nottingham Power Rig (Bassey and Short, 1990) was built to measure neuromuscular function of the leg extensors. The force output was transmitted via a foot plate, lever and chain, which was fixed onto a motor-driven freewheel body and hub (Dura-Ace FH-7700, Shimano ${ }^{\circledR}$ Inc., Osaka, Japan). The technical details of the isokinetic dynamometer have been previously described (Koninckx et al., 2008). Briefly, the rotation speed of the lever arm was synchronized with the servo-controlled rotation of the motor axle (CM71 motor type combined with a Movidrive controller MDS60A, SEW-Eurodrive, Bruchsal, Germany) using a timing belt and two identical pulleys (Synchroforce HTD 925-5M CXP, Contitech, Germany). Torque values were measured by a torque transducer with an overall accuracy of $<0.25 \%$ (1703 series, Lebow ${ }^{\circledast}$ Products Inc., Troy, United States). A dSpace interface (dSpace-GmBH, Paderborn, Germany) was used for data streaming at $1000 \mathrm{~Hz}$, with a real-time control program (Simulink, The Mathworks Inc., Natick, United States). Movement of the lever arm was initiated by surpassing a cut-off torque of $20 \%$ of maximal isometric single-joint strength inducing a low-level of pretension (i.e. $20-70 \mathrm{Nm}$ ) as previously recommended (Tillin et al., 2013). Measurements were performed unilaterally on the right side. Subjects were seated on a backward-inclined $\left(5^{\circ}\right)$ chair, which was vertically and horizontally adjustable. In addition, the lever arm was adjustable to allow standardization of the multi-joint range of motion. Range of motion was set from a knee joint angle of $90^{\circ}$ to $160^{\circ}$ and from a hip joint angle of $70^{\circ}$ to $115^{\circ}$, with a fully extended leg corresponding to a knee and hip angle of $180^{\circ}$ (Fig. 1). The hips and shoulders were stabilized with safety belts. The right foot was fully supported and fixed

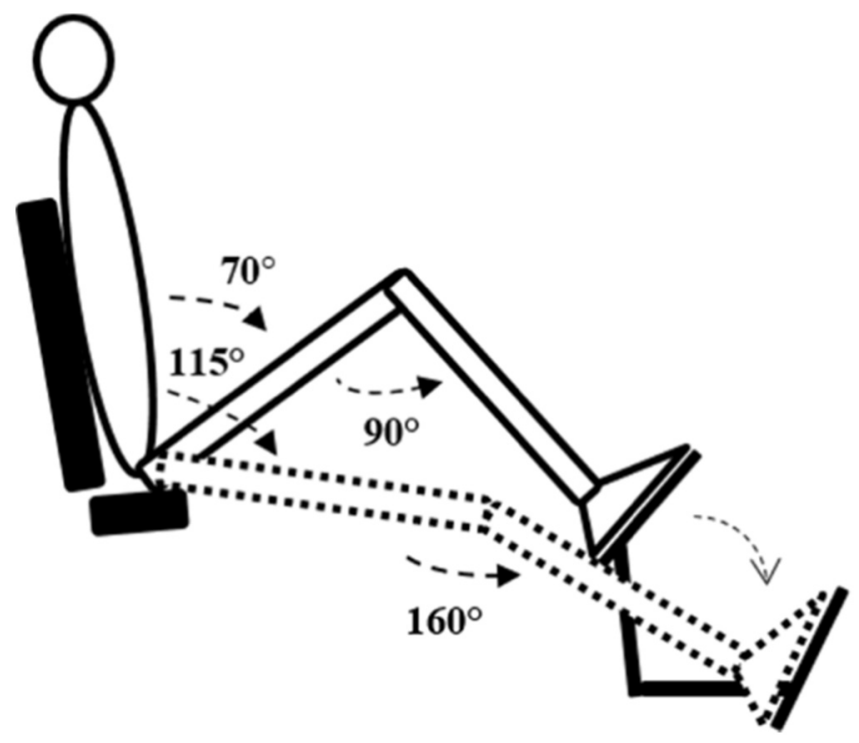

Fig. 1. Start and end position of the multi-joint leg press movement. 
to the foot plate using a solid strap with the lateral malleolus aligned with the point of force application, provoking a heel thrust to minimize the influence of lower leg muscles and ankle movement. Subjects wore the same flat non-cushioning shoes to minimize the cushioning effect during the initial impact of the rapid contractions.

During the tests, subjects were asked to fold their hands across the chest to avoid extraneous movements. They performed maximal leg press movements while lever arm angle speed was held constant at $180^{\circ} / \mathrm{s}, 300^{\circ} / \mathrm{s}$ and $420^{\circ} / \mathrm{s}$. Even though absolute angular velocities of multi- and single-joint tests differ extensively (i.e. read 60, 180 and $300^{\circ} / \mathrm{s}$ versus $180^{\circ} / \mathrm{s}, 300^{\circ} / \mathrm{s}$ and $420^{\circ} / \mathrm{s}$ ), tests were performed at similar knee angular velocities (i.e. 60,180 and $300^{\circ} / \mathrm{s}$ in single-joint versus $\sim 115,191$ and $267^{\circ} / \mathrm{s}$ in multi-joint tests). Knee angular velocities were calculated as the knee joint's range of motion over the time of the particular tests. Each condition was performed three times with $30 \mathrm{~s}$ rest periods in between. In between different velocity conditions, participants were allowed to rest for 2 min to minimize fatigue. During each test, the subjects were clearly instructed by the test leader to perform the tests as fast and hard as possible.

\subsection{Functional performance testing}

Squat jumps were assessed as a measure of functional performance allowing maximal neuromuscular performance in a similar knee range of motion as the isokinetic test methods, while avoiding the ceiling effects that are often reported in standard functional performance test batteries. The squat jumps were performed on a force platform (OR6-7, Advanced Mechanical Technology Inc., Watertown, United States) at a sampling frequency of $1000 \mathrm{~Hz}$. Subjects kept their hands placed on the hips to avoid the effect of arm-swing on jump performance. Subjects were instructed to start in a squat position at $90^{\circ}$ knee angle and jump as high as possible without performing any countermovement. All trials that were preceded by a countermovement (i.e. ground reaction force $<0.015 *$ body mass) were excluded. After a first trial, three jumps were performed with $30 \mathrm{~s}$ rest in between.

\subsection{Signal processing}

All signals were processed off-line using a commercial software package (Matlab R2016b, The MathWorks Inc., Natick, United States). Torque signals of the single- and multi-joint tests were filtered using a fourth-order low-pass Butterworth filter with a $10 \mathrm{~Hz}$ and $20 \mathrm{~Hz}$ cut-off frequency, respectively. Instantaneous power (Watt) was calculated as the product of torque $(\mathrm{Nm})$ and velocity $(\mathrm{rad} / \mathrm{s})$. It should be noted that due to the isokinetic nature of the present measurements, power production represents the dynamic torque production at standardized movement velocities. Peak power (Ppeak, Watt) was determined as the highest value of the power curve. The rate of power development (RPD, Watt/s) was calculated as the linear slope of the power-time curve from the start of the movement till isokinetic speed was reached. The start of the movement was determined as the point where the acceleration reached a threshold of $150^{\circ} / \mathrm{s}^{2}$ for the single-joint tests (Van Driessche et al., 2018a) and where the cut-off torque was reached for the multijoint tests. The tests with the highest Ppeak were used for further analyses. Reliability of maximal and rapid muscle characteristics of the knee extensors using isokinetic dynamometry have been previously reported (ICC: 0.85-0.96; SEM(\%): 3.6-12.5) (Brown et al., 2005; Van Driessche et al., 2018b). Reliability of the multi-joint measurements was assessed in 16 older adults, who returned for a third session to repeat the multi-joint tests (ICC: 0.87-0.97; SEM(\%): 4-18.7).

Ground reaction force signals of the squat jump tests allowed for the determination of body center of mass (BCM) velocity at take-off and hence the calculation of jump height, according to previous procedures (Edwen et al., 2014). The highest jump performance was used for further analyses.

\subsection{Statistical analyses}

All statistical analyses were performed using $\mathrm{R}$ software, version 1.0.153 (R_Core_Team, 2017). To study the effects of age and test method on rapid and maximal neuromuscular function, we built linear mixed models for Ppeak and RPD using the function lmer provided by the R-package lme4 (Bates et al., 2015). The response variable in all models was log-transformed to make relative comparison of the agerelated declines between speeds and test methods possible (Törnqvist et al., 1985). Age and test method (single- or multi-joint) were entered as fixed effects into the model. The variable age was used as continuous variable. In addition to the fixed-effects terms, the models included subject as a random effect to correct for the repeated measures design. The effect of covariates such as speed, sex, body mass, lever arm length (only for the multi-joint tests) and their possible interactions were analyzed in a series of separate linear mixed effects models using stepup model comparisons. This iterative model fitting procedure started with the basic model of investigating the effect of age and test method on the response variable, then sequentially adding predictor terms. The Akaike information criterion (AIC) and a likelihood ratio test, performing the R-function 'anova', were used to compare models. For all models we tested underlying assumptions (e.g. normality and homogeneity of residuals) by visual inspection of residual plots. We found that no assumption was violated. After fitting the models, model estimates of the log-transformed data were exponentiated for interpretability of the relative slopes (i.e. annual decline rates in percent). $P$ values were obtained using the R-package lmerTest (Kuznetsova et al., 2016).

Post hoc, to test multi-versus single-joint neuromuscular function at similar speeds, we fitted a new model in which speed was modeled as a categorical variable. As fixed effects, we entered age and speed. Sex and body mass were added as covariates. Subject was included as random effect. The R-function 'relevel' was used in three separate models to compare multi-joint $115^{\circ} / \mathrm{s}$ with single-joint $60^{\circ} / \mathrm{s}$, multi-joint $191^{\circ} / \mathrm{s}$ with single-joint $180^{\circ}$ /s and multi-joint $267^{\circ}$ /s with single-joint $300^{\circ} / \mathrm{s}$.

Pearson's correlation coefficients were calculated to examine the relationship of multi- versus single-joint power measures with squat jump performance. Fischer $\mathrm{z}$ transformation was used to statistically compare correlation coefficients. Statistical significance was set at $p<.05$ for all analyses.

\section{Results}

Mixed-effects model comparisons revealed significant main effects for age, test method, speed, sex and body mass for both response variables (Table 1). The estimate of the intercept in Table 1 can be interpreted as the reference value (i.e. man of 20 years old that is tested multi-joint at the lowest speed) in $\log$ (Watt) for Ppeak and $\log \left(\mathrm{Nm} / \mathrm{s}^{2}\right)$ for RPD. In line with this, the estimate of age, test method, speed, sex and body mass can be interpreted as the rate of change in Ppeak and RPD per unit of the independent variable. Lever arm length was first included to test its possible performance enhancing effect during the multi-joint tests in comparison with the single-joint tests, but was removed from the model because it was not significant. A significant interaction effect between age and test method was found for Ppeak and RPD, indicating that Ppeak declines more in the single-joint action whereas RPD declines more in the multi-joint action during ageing (Table 2, Fig. 2). No significant interaction effects of age with speed, sex or body mass were found.

The comparison of the age-related decline in multi- and single-joint Ppeak at similar speeds revealed significantly lower declines in multijoint tests. Interestingly, age-related declines in RPD were higher in multi-joint than single-joint tests at low and moderate speed.

Both single- and multi-joint tests were positively correlated with squat jump performance. Interestingly, the association between squat jump height and RPD multi-joint was significantly higher than the 
Table 1

Results of the linear mixed effects models.

\begin{tabular}{llll}
\hline Term & Estimate & SE & $p$-Value \\
\hline Ppeak & & & \\
Intercept & 5.618 & 0.134 & $<0.001$ \\
Age & -0.008 & 0.001 & $<0.001$ \\
Method & -0.667 & 0.033 & $<0.001$ \\
Speed & 0.004 & 0.000 & $<0.001$ \\
Sex & -0.276 & 0.038 & $<0.001$ \\
BM & 0.010 & 0.001 & $<0.001$ \\
Age $*$ method & -0.003 & 0.001 & $<0.001$ \\
RPD & & & $<0.001$ \\
Intercept & 7.091 & 0.289 & $<0.001$ \\
Age & -0.019 & 0.002 & $<0.001$ \\
Method & -0.483 & 0.096 & $<0.001$ \\
Speed & 0.004 & 0.000 & $<0.001$ \\
Sex & -0.414 & 0.081 & 0.0122 \\
BM & 0.015 & 0.003 & \\
Age $*$ method & 0.005 & 0.002 & \\
\hline
\end{tabular}

Data represent the effect of age and test method in interaction, speed, sex and body mass $(\mathrm{BM})$ as fixed factors and subject $(n=96)$ as random factor on maximal power (Ppeak) and rate of power development (RPD). Estimates $=$ change in Ppeak $(\log ($ Watt $))$ and RPD $\left(\log \left(\mathrm{Nm} / \mathrm{s}^{2}\right)\right)$ per unit of the respective term compared to the intercept, $\mathrm{SE}=$ standard error, * refers to an interaction term.

Table 2

Annual decline rates in peak power and rate of power development.

\begin{tabular}{llll}
\hline Method & Speed & Ppeak (\%) & RPD (\%) \\
\hline \multirow{2}{*}{ Single-joint } & Overall & -1.04 & -1.42 \\
& Low & -0.93 & -1.32 \\
& Moderate & -1.06 & -1.44 \\
Multi-joint & High & -1.14 & -1.51 \\
& Overall & $-0.77^{*}$ & $-1.92^{*}$ \\
& Low & $-0.70^{*}$ & $-1.95^{*}$ \\
& Moderate & $-0.77^{*}$ & $-2.04^{*}$ \\
& High & $-0.84^{*}$ & -1.80
\end{tabular}

Data are exponentiated estimates from mixed model analyses, which express the annual age-related decline rates in percent for maximal power (Ppeak) and rate of power development (RPD) in single-joint tests overall, at $60^{\circ} / \mathrm{s}$ (low), $180 \%$ s (moderate) and $300^{\circ} / \mathrm{s}$ (high speed) and multi-joint tests overall, at $115 \%$ s (low), $191 \%$ s (moderate) and $267^{\circ} / \mathrm{s}$ (high speed). $*=$ significantly different from single-joint $(p<.05)$.

association between squat jump height and RPD single-joint (Table 3). For Ppeak, multi- and single-joint tests were equally related to squat jump performance.

\section{Discussion}

This study investigated the effect of ageing on maximal and rapid muscle characteristics in dynamic single- and multi-joint actions across the adult life span. In line with our first hypothesis, leg extension RPD $(-1.42$ to $-1.92 \%)$ declines more during ageing than Ppeak $(-1.04$ to $-0.77 \%$ ) for both single- and multi-joint movements. In accordance to our second and third hypotheses, RPD in multi-joint movements declines more during ageing and is more strongly related to squat jump performance than RPD in single-joint movements. These observations emphasize the striking age-related decline in rapid muscle characteristics, especially in multi-joint leg extension movements.

Surprisingly, this study showed a greater age-related decline of Ppeak in single- versus multi-joint actions. Across all speeds, Ppeak showed an average decline rate of $0.77 \%$ per year in the multi-joint mode, whereas Ppeak in the single-joint mode showed a significantly greater average decline rate of $1.04 \%$ per year (Table 1 ). The magnitude of these annual decline rates are similar as previously described for single-joint (i.e. 0.7-1.8\%) (Kennis et al., 2014; Lanza et al., 1985) and multi-joint tests (i.e. 0.6-1.5\%) (Kostka, 2005; Yamauchi et al., 2009; Macaluso and De Vito, 2003; Allison et al., 2013; Edwen et al., 2014). Although, to our knowledge, no previous research compared the agerelated declines in power output between single- and multi-joint test methods, it has previously been suggested that the cooperative work of multiple muscles working over multiple joints could lead to a compensation strategy to prevent for the reduced performance output at older age (Hortobágyi et al., 2003). These findings suggest that the greater age-related decline of Ppeak in single- versus multi-joint actions found in this study may highlight the adaptive mechanical plasticity of the ageing neuromuscular system. In this way, multi-joint testing could underestimate the age-related declines in maximal power production, which is in favor of single-joint testing.

However, maximal power may not be representative for the overall decline in neuromuscular function. To illustrate, previous studies have emphasized the importance of the initial phase of rapid single-joint contractions by demonstrating greater age-related declines in RFD and RPD compared to maximal strength and power (Thompson et al., 2013; Van Driessche et al., 2018a). In general, the effect of age on the neuromuscular level refers to a combination of neural and muscular changes. It has been shown that RFD is predominantly influenced by neural characteristics next to type II myofibre content, especially in older adults (Klass et al., 1985). Muscular properties become more relevant in later stages of rapid muscle contractions ( $>75-150 \mathrm{~ms}$ ) in contrast with the neural input that predetermines the first $75 \mathrm{~ms}$ (Folland et al., 2014; Maffiuletti et al., 2016). Considering that Ppeak was generally reached after $>150 \mathrm{~ms}$ across speeds over the entire adult life span in the current study, compensatory actions of synergist muscles could have been initiated by the neural system in the multijoint tests and could partly have masked the effect of age on the neuromuscular changes that are reflected in the single-joint tests. Whereas short response times (i.e. $<75 \mathrm{~ms}$ ) may limit the ability to initiate compensatory muscle actions in multi-joint performance, resulting in greater age-related declines. Therefore, we hypothesized that RPD would decline more than Ppeak. The results of the present study confirm that this is true for single-joint contractions, but in particular also for multi-joint leg-extension tests.

The variable RPD is representative for the initial acceleration phase (i.e. first 50-75 ms). In line with our second hypothesis, the present study showed a greater age-related decline of RPD in multi- $(-1.92 \%)$ versus single-joint $(-1.42 \%)$ actions. This greater age-related decline in multi-joint was apparent at slow and moderate speeds, but not at high speed. The latter can be explained by the longer time that was needed to reach the higher isokinetic speeds (i.e. $\sim 75 \mathrm{~ms}$ ). The longer the time to develop power, the higher the chance that the complex coordination pattern of agonist and synergist muscles has been well initiated and that compensatory actions of synergist muscles might have led to an underestimation of the age-related deterioration of neuromuscular function in multi-joint movement. Although no previous research focused on rapid power characteristics across the adult life span in single- versus multi-joint movements, recent reports compared rapid strength characteristics between age-groups in multi-joint movements. Izquierdo et al. reported a $46 \%$ lower peak force and a $64 \%$ lower peak RFD for older men ( $~ 70$ years) compared with young men ( $\sim 20$ years) performing an isometric bilateral squat (Izquierdo et al., 1999). Allison et al. only found a $19 \%$ lower peak force and a $21 \%$ lower peak RFD between young ( $\sim 24$ years) and older ( $~ 66$ years) men performing an isometric unilateral leg press movement (Allison et al., 2013). Surprisingly, the latter did not find an effect of age on the rapid force production during the initial phase (i.e. first $100 \mathrm{~ms}$ ) of a multijoint isometric contraction, suggesting that older men and young men have a similar ability to activate the involved muscles during rapid efforts. However, the more extended position and initial pretension in their measurements $(\sim 150 \mathrm{~N})$ might explain their different results. Furthermore, both aforementioned studies did not perform single- and multi-joint tests within the same cohort, making comparison between 


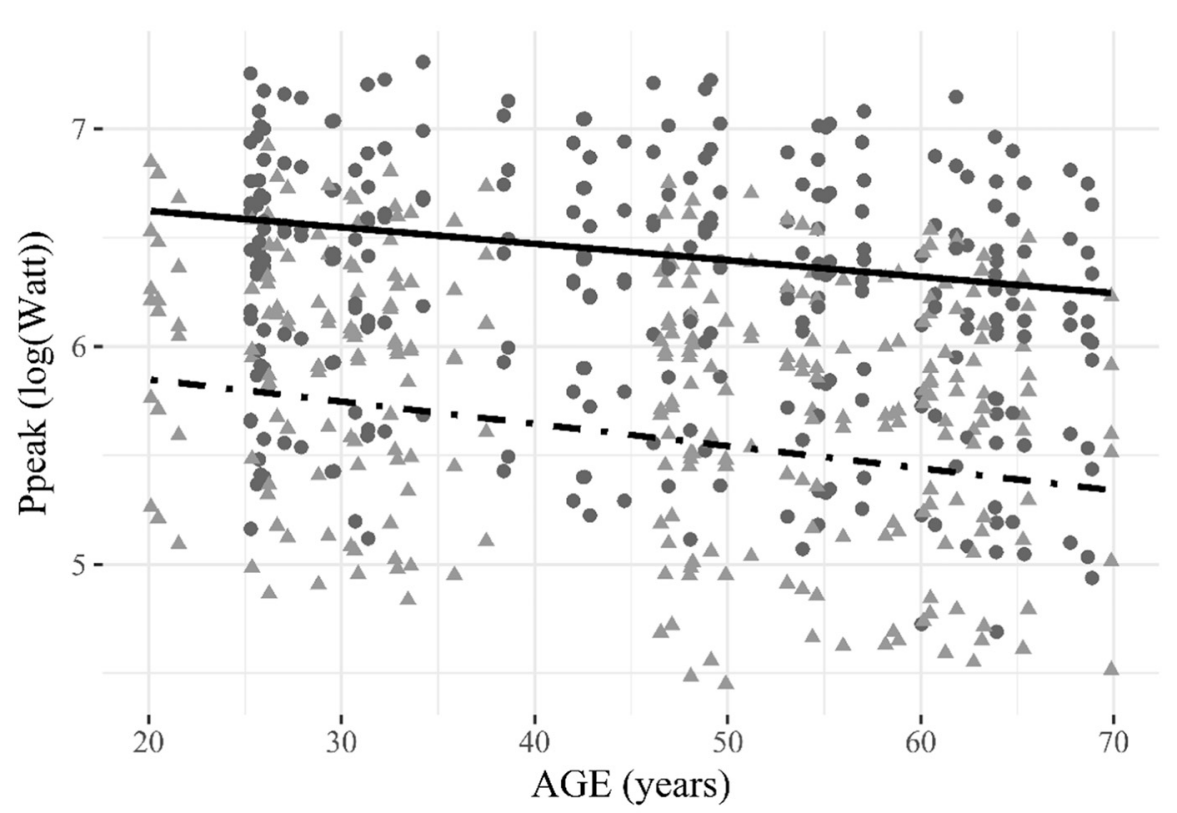

B) MEN $\triangle$ WOMEN - Multi-joint $\cdot-$ Single-joint

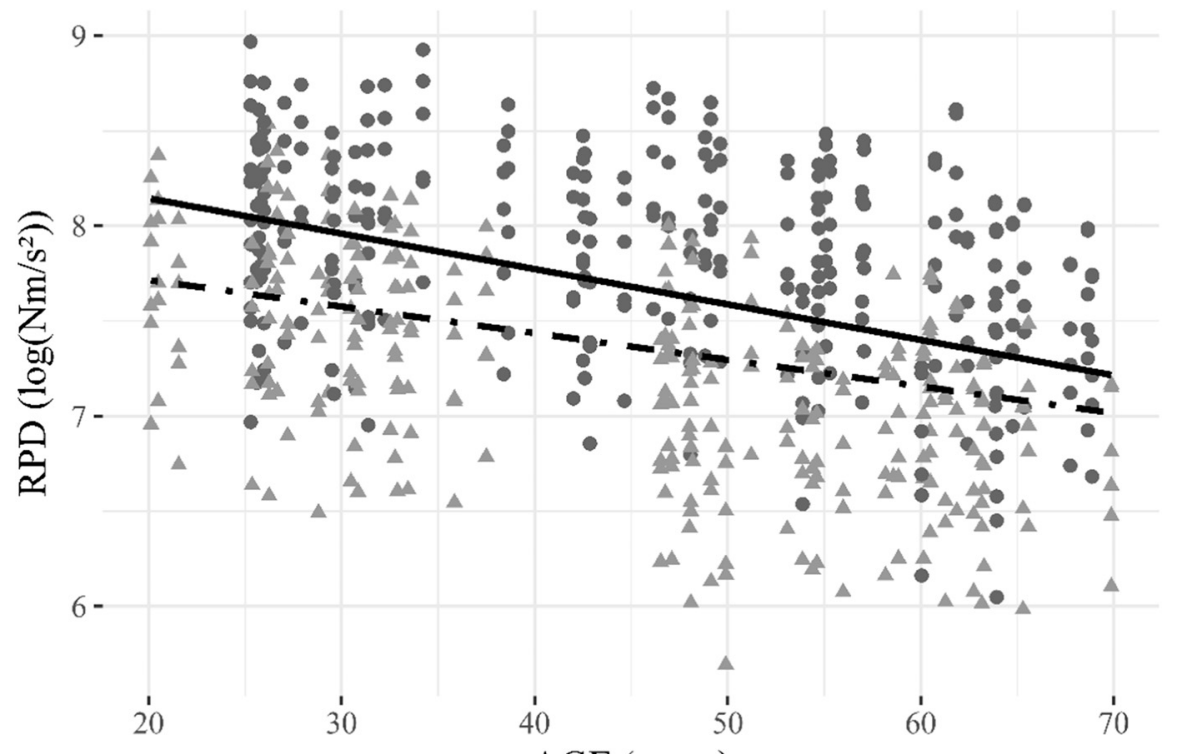

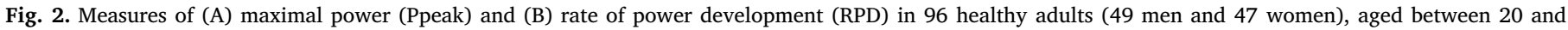

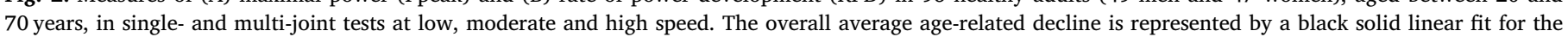
multi-joint tests and a black dotdashed linear fit for the single-joint tests. Data are displayed on a log scale.

methods difficult. Only recently, Thompson et al. demonstrated greater age-related reductions of RFD in multi-joint versus single-joint actions within the same cohort (Thompson et al., 2018). They suggested that single-joint static strength tests may underestimate age-related rapid strength impairments, which is in line with our findings of rapid power (RPD) characteristics in dynamic tests.

At this point, we can only speculate on the relative contribution of neural and muscular determinants of RPD. However, our results suggest that the effect of age on the neural drive of muscle action has a detrimental role on power development during very fast actions, especially in multi-joint movements compared to single-joint movements. This is especially relevant at older age, considering that most daily activities include multi-joint movements and failure to perform fast actions can lead to falling and fractures. In the current study, squat jump height was highly related to Ppeak in single- and multi-joint movements. According to our third hypothesis, jump height was more strongly related to RPD in multi-joint compared to single-joint movements, indicating the functional relevance of the multi-joint test methodology.

A potential methodological limitation of the present study was the isokinetic test approach. Isokinetic tests can be considered as less functional compared to isotonic tests because of their speed controlling nature. This speed control could have led to an underestimation of 
Table 3

Association between squat jump performance and Ppeak or RPD.

\begin{tabular}{llll}
\hline Method & Speed & Ppeak/BM & RPD/BM \\
\hline \multirow{2}{*}{ Single-joint } & Low & 0.68 & 0.44 \\
& Moderate & 0.74 & 0.50 \\
\multirow{3}{*}{ Multi-joint } & High & 0.77 & 0.61 \\
& Low & 0.80 & $0.77^{*}$ \\
& Moderate & 0.82 & $0.80^{*}$ \\
& High & 0.85 & $0.82^{*}$ \\
\hline
\end{tabular}

Data are Pearson's correlation coefficients ( $r$-values) for the association of maximal power (Ppeak) and rate of power development (RPD) relative to body mass (BM) measured in single-joint tests at $60^{\circ} / \mathrm{s}$ (low speed), $180^{\circ} / \mathrm{s}$ (moderate speed) and $300 \%$ s (high speed) and in multi-joint tests at $115^{\circ} / \mathrm{s}$ (low speed), $191 \%$ s (moderate speed) and $267^{\circ} / \mathrm{s}$ (high speed) with jump height measured in squat jump tests in healthy adults $(n=96)$ aged between 20 and 70 years. All correlation coefficients were statistically significant $(p<.05)$. * = significantly more strongly associated with squat jump performance compared to single-joint tests.

power compared to non-isokinetic approaches (Aagaard et al., 1994). Furthermore, differences in machine control between clinical/commercial (i.e. Biodex) and custom made equipment could have influenced our results. Though, the use of this approach enabled the comparison of single- and multi-joint movements at similar predetermined speeds. Next, the cross-sectional design of this study may be confounded by inter individual differences and age-related declines may be different in a longitudinal design. Furthermore, the oldest subject was 69 years old. Although we found large age-related declines in neuromuscular function in our sample of adults between 20 and 69 years of age, we are not able to extrapolate our results to ages over 70 years. To note, an accelerated deterioration of power output after the age of 65 was previously reported with decline rates of $3-4 \%$ per year, which emphasizes the age-related deterioration of neuromuscular function at older age (Skelton et al., 1994). However, we believe that it is important to explore the early age-related declines in neuromuscular function to allow for appropriate screening and prevention methods before daily life functioning becomes limited.

To conclude, for the first time multi-joint leg extension tests were compared to single-joint tests in terms of rapid power characteristics across the adult life span. The key finding is that RPD declines more in multi-joint than in single-joint actions during ageing. Although the majority of research and clinical practice is focused on single-joint testing and maximal output parameters like Ppeak, this study indicated that ageing has an even more detrimental effect on the initial phase of rapid power development (RPD) especially in multi-joint tests. Moreover, multi-joint RPD was shown to be strongly associated with functional performance. Given that many daily life activities include fast multi-joint movements, multi-joint RPD can be considered relevant for daily life functional performance. Therefore, these findings may be of high importance for the detection of functional disability at older age. Whether training interventions targeting gains in multi-joint RPD can lead to prevention of functional disability should be further investigated. Finally, the mechanisms behind the age-related decline in RPD in multi-joint actions should be investigated in future research.

\section{Acknowledgements}

The authors gratefully acknowledge the participants of the present study for their time and efforts, physiotherapists Maarten Thaens and Koen Swinnen for their assistance during the measurements, as well as mechanical specialist Peter Verstraeten and electronic engineer Paul Meugens for their help and technical advice in the construction of our custom-made leg-press machine.

\section{References}

Aagaard, P., Simonsen, E.B., Trolle, M., Bangsbo, J., Klausen, K., 1994. Moment and power generation during maximal knee extensions performed at low and high speeds. Eur. J. Appl. Physiol. Occup. Physiol. 69 (5), 376-381.

Aagaard, P., Suetta, C., Caserotti, P., Magnusson, S.P., Kjær, M., 2010. Role of the nervous system in sarcopenia and muscle atrophy with aging: strength training as a countermeasure. Scand. J. Med. Sci. Sports 20 (1), 49-64.

Allison, S.J., Brooke-Wavell, K., Folland, J.P., 2013. Multiple joint muscle function with ageing: the force-velocity and power-velocity relationships in young and older men. Aging Clin. Exp. Res. 25 (2), 159-166.

Azegami, M., Ohira, M., Miyoshi, K., Kobayashi, C., Hongo, M., Yanagihashi, R., et al., 2007. Effect of single and multi-joint lower extremity muscle strength on the functional capacity and ADL/IADL status in Japanese community-dwelling older adults. Nurs. Health Sci. 9 (3), 168-176.

Bassey, E.J., Short, A.H., 1990. A new method for measuring power output in a single leg extension: feasibility, reliability and validity. Eur. J. Appl. Physiol. Occup. Physiol. 60 (5), 385-390.

Bates, D., Mächler, M., Bolker, B., Walker, S., 2015. Fitting linear mixed-effects models using lme4. J. Stat. Softw. 67 (1), 48.

Billot, M., Duclay, J., Simoneau-Buessinger, E.M., Ballay, Y., Martin, A., 2014. Is cocontraction responsible for the decline in maximal knee joint torque in older males? Age (Dordr.) 36 (2), 899-910.

Brown, L.E., Whitehurst, M., Findley, B.W., 2005. Reliability of rate of velocity development and phase measures on an isokinetic device. J. Strength Cond. Res. 19 (1), 189-192.

Clark, D.J., Manini, T.M., Fielding, R.A., Patten, C., 2013. Neuromuscular determinants of maximum walking speed in well-functioning older adults. Exp. Gerontol. 48 (3), 358-363.

Cuoco, A., Callahan, D.M., Sayers, S., Frontera, W.R., Bean, J., Fielding, R.A., 2004. Impact of muscle power and force on gait speed in disabled older men and women. J. Gerontol. A Biol. Sci. Med. Sci. 59 (11), 1200-1206.

Edwen, C.E., Thorlund, J.B., Magnusson, S.P., Slinde, F., Svantesson, U., Hulthen, L., et al., 2014. Stretch-shortening cycle muscle power in women and men aged 18-81 years: influence of age and gender. Scand. J. Med. Sci. Sports 24 (4), 717-726.

Foldvari, M., Clark, M., Laviolette, L.C., Bernstein, M.A., Kaliton, D., Castaneda, C., et al., 2000. Association of muscle power with functional status in community-dwelling elderly women. J. Gerontol. A Biol. Sci. Med. Sci. 55 (4) M192-M9.

Folland, J.P., Buckthorpe, M.W., Hannah, R., 2014. Human capacity for explosive force production: neural and contractile determinants. Scand. J. Med. Sci. Sports 24 (6), 894-906.

Goodpaster, B.H., Park, S.W., Harris, T.B., Kritchevsky, S.B., Nevitt, M., Schwartz, A.V., et al., 2006. The loss of skeletal muscle strength, mass, and quality in older adults: the health, aging and body composition study. J. Gerontol. A Biol. Sci. Med. Sci. 61 (10), 1059-1064.

Häkkinen, K., Newton, R.U., Gordon, S.E., McCormick, M., Volek, J.S., Nindl, B.C., et al., 1998. Changes in muscle morphology, electromyographic activity, and force production characteristics during progressive strength training in young and older men. J. Gerontol. A Biol. Sci. Med. Sci. 53A (6) B415-B23.

Hortobágyi, T., Mizelle, C., Beam, S., DeVita, P., 2003. Old adults perform activities of daily living near their maximal capabilities. J. Gerontol. A Biol. Sci. Med. Sci. 58 (5) M453-M60.

Izquierdo, M., Aguado, X., Gonzalez, R., Lopez, J.L., Hakkinen, K., 1999. Maximal and explosive force production capacity and balance performance in men of different ages. Eur. J. Appl. Physiol. Occup. Physiol. 79 (3), 260-267.

Kennis, E., Verschueren, S., Van Roie, E., Thomis, M., Lefevre, J., Delecluse, C., 2014. Longitudinal impact of aging on muscle quality in middle-aged men. Age (Dordr.) 36 (4), 9689.

Klass, M., Baudry, S., Duchateau, J., 1985. Age-related decline in rate of torque development is accompanied by lower maximal motor unit discharge frequency during fast contractions. J. Appl. Physiol. 104 (3), 739-746 2008.

Koninckx, E., van Leemputte, M., Hespel, P., 2008. Effect of a novel pedal design on maximal power output and mechanical efficiency in well-trained cyclists. J. Sports Sci. 26 (10), 1015-1023.

Kostka, T., 2005. Quadriceps maximal power and optimal shortening velocity in 335 men aged 23-88 years. Eur. J. Appl. Physiol. 95 (2-3), 140-145.

Kuznetsova, A., Brockhoff, P.B., Christensen, R.H.B., 2016. lmerTest: Tests in Linear Mixed Effects Models. R Package Version 2.0-33.

Lanza, I.R., Towse, T.F., Caldwell, G.E., Wigmore, D.M., Kent-Braun, J.A., 1985. Effects of age on human muscle torque, velocity, and power in two muscle groups. J. Appl, Physiol. 95 (6), 2361-2369 2003.

Macaluso, A., De Vito, G., 2003. Comparison between young and older women in explosive power output and its determinants during a single leg-press action after optimisation of load. Eur. J. Appl. Physiol. 90 (5), 458-463.

Madigan, M.L., Lloyd, E.M., 2005. Age and stepping limb performance differences during a single-step recovery from a forward fall. J. Gerontol. A Biol. Sci. Med. Sci. 60 (4), 481-485.

Maffiuletti, N.A., Aagaard, P., Blazevich, A.J., Folland, J., Tillin, N., Duchateau, J., 2016. Rate of force development: physiological and methodological considerations. Eur. J. Appl. Physiol. 116 (6), 1091-1116.

Pijnappels, M., Bobbert, M.F., van Dieën, J.H., 2005. Control of support limb muscles in recovery after tripping in young and older subjects. Exp. Brain Res. 160 (3), 326-333.

R_Core_Team, 2017. R: A Language and Environment for Statistical Computing. R Foundation for Statistical Computing, Vienna, Austria.

Skelton, D.A., Greig, C.A., Davies, J.M., Young, A., 1994. Strength, power and related 
functional ability of healthy people aged 65-89 years. Age Ageing 23 (5), 371-377. Thompson, B.J., Ryan, E.D., Sobolewski, E.J., Conchola, E.C., Cramer, J.T., 2013. Age related differences in maximal and rapid torque characteristics of the leg extensors and flexors in young, middle-aged and old men. Exp. Gerontol. 48 (2), 277-282.

Thompson, B.J., Whitson, M., Sobolewski, E.J., Stock, M.S., 2018. Effects of age, joint angle, and test modality on strength production and functional outcomes. Int. J. Sports Med. 39 (2), 124-132.

Tillin, N.A., Pain, M.T., Folland, J., 2013. Explosive force production during isometric squats correlates with athletic performance in rugby union players. J. Sports Sci. 31 (1), 66-76.

Törnqvist, L., Vartia, P., Vartia, Y.O., 1985. How should relative changes be measured?
Am. Stat. 39 (1), 43-46.

Van Driessche, S., Delecluse, C., Bautmans, I., Vanwanseele, B., Van Roie, E., 2018a. Agerelated differences in rate of power development exceed differences in peak power. Exp. Gerontol. 101, 95-100.

Van Driessche, S., Van Roie, E., Vanwanseele, B., Delecluse, C., 2018b. Test-retest reliability of knee extensor rate of velocity and power development in older adults using the isotonic mode on a Biodex system 3 dynamometer. PLoS One 13 (5), e0196838. Yamauchi, J., Mishima, C., Nakayama, S., Ishii, N., 2009. Force-velocity, force-power relationships of bilateral and unilateral leg multi-joint movements in young and elderly women. J. Biomech. 42 (13), 2151-2157. 